Check for updates

Cite this: RSC Adv., 2019, 9, 30803

Received 7th August 2019

Accepted 16th September 2019

DOI: 10.1039/c9ra06130d

rsc.li/rsc-advances

\title{
An injectable molecular hydrogel assembled by antimicrobial peptide PAF26 for antimicrobial application
}

\author{
Fengyi Cao, (D)* Lin Mei, (D) Genxing Zhu, (D) Meng Song and Xueli Zhang
}

\begin{abstract}
Wound infection is a crucial factor that inhibits wound recovery. A feasible measure to solve this problem is using antimicrobial biomaterials to suppress the microbial growth. In this work, an amphipathic antimicrobial peptide (Ac-RKKWFW-NH2, PAF26) was investigated to form the antimicrobial hydrogel. Triggered by $\mathrm{pH}$, PAF26 peptide could self-assemble into a hydrogel, and the hydrogel formed was injectable and exhibited shear-thinning ability. Antimicrobial experiments demonstrated that the selfassembled hydrogel had an outstanding antimicrobial ability against pathogenic microbes such as Candida albicans, Staphylococcus aureus, and Escherichia coli via destroying the cell membrane structure. Thus, this study provides a novel method for preparing an injectable antimicrobial peptide hydrogel for antimicrobial therapies.
\end{abstract}

\section{Introduction}

In the past decades, new kinds of antimicrobial wound dressings, such as functional cotton gauzes, ${ }^{1,2}$ hydrocolloids, ${ }^{3,4}$ and hydrogels, ${ }^{5-7}$ have been studied for inhibiting wound infections. Among them, hydrogels, due to their high swelling ratio, are the most promising biomaterial for accelerating the wound healing process. ${ }^{8}$ The hydrogel is a kind of biomaterial, which is made from natural or artificial polymers. However, its antimicrobial ability is not satisfied. To improve the antimicrobial ability, antimicrobial agents, including antimicrobial drugs, ${ }^{\mathbf{9}, 10}$ metal ions (such as $\mathrm{Ag}^{+}, \mathrm{Zn}^{2+}$, and $\mathrm{Cu}^{2+}$ ), ${ }^{11-13}$ or antimicrobial peptides, ${ }^{14,15}$ have been introduced.

Antimicrobial peptides (AMPs), primarily present in silkworms, are an important class of components for protecting the host against microbial infections. By now, it has been widely found in natural organisms, and more than 1200 different AMPs are known. ${ }^{16}$ AMPs are reported to exhibit wide spectrum of antibacterial ability, good biocompatibility, and low drugresistance, ${ }^{17,18}$ which made them potential candidates as antimicrobial agents. Generally, AMPs have a length of 4-100 amino acids with amphipathic structure, and most are cationic peptides.

The molecular hydrogel, a novel kind of hydrogel, has been extensively studied in recent years due to its big potential for cell culture, drug delivery, and regenerative medicine. ${ }^{19-21}$ Unlike conventional hydrogel, the molecular hydrogel is formed by a molecular self-assembly in water, without the process of

School of Materials and Chemical Engineering, Zhongyuan University of Technology, Zhengzhou 450007, P. R. China. E-mail: caofengyi0513@126.com; Tel: +86 371 69975784 cross-linking or drug loading. Moreover, the molecular hydrogel has the ability of self-delivery and could realize a higher local density at the target sites. The self-assembled molecules include drugs, ${ }^{22}$ peptides, ${ }^{23-25}$ sugar-based compounds, ${ }^{26}$ and amino acid-derivatives, ${ }^{27}$ which could work as both hydrogelator and active "drug". For example, Yang and his group ${ }^{28}$ used two kinds of anti-inflammatory molecules and a uranyl ion chelating ligand to form the supramolecular hydrogel. The hydrogel could self-deliver and be used for simulated uranium wound treatments.

In this study, a hexapeptide PAF26 (Scheme 1) was observed to form the self-assembled molecular hydrogel. With the amphipathic structure, the cationic peptide could self-assemble into a molecular hydrogel triggered by $\mathrm{pH}$. Due to the antimicrobial ability of PAF26 peptide, ${ }^{29,30}$ when contacted with pathogenic microbes, the hydrogel could destroy the cell-wall and kill the microbes.

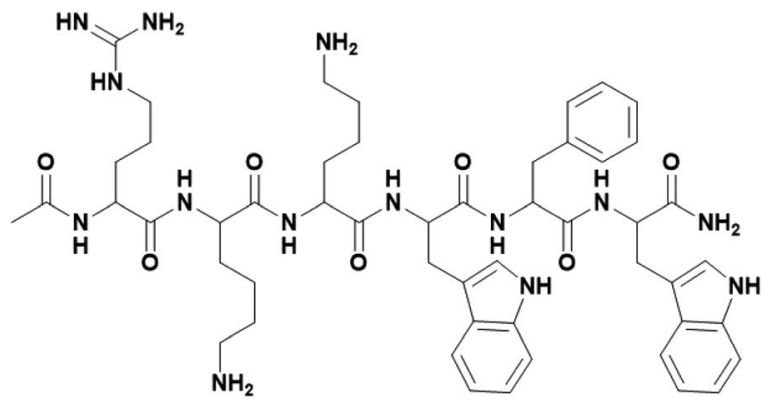

Scheme 1 Chemical structure of PAF26 peptide: Ac-RKKWFW$\mathrm{NH}_{2}$. 


\section{Experimental section}

\section{Reagents and materials}

The PAF26 peptide: Ac-RKKWFW- $\mathrm{NH}_{2}$ (molecular weight: $991.19 \mathrm{~g} \mathrm{~mol}^{-1}$, GC purity $\geq 95 \%$ ) was purchased from Shanghai GL Biochem Ltd. (Shanghai, China). Sodium hydroxide (AR) was purchased from Sinopharm chemical reagent Co., Ltd. (Shanghai, China). Potassium bromide (SP) was purchased from Shanghai Yi En chemical technology Co., Ltd. Candida albicans (ATCC10231), Staphylococcus aureus (ATCC6538), and Escherichia coli (ATCC25922) were purchased from Shanghai Luwei technology company (Shanghai, China). Sabouraud dextrose broth (SDB), sabouraud dextrose agar (SDA), casein soya bean digest broth (TSB), and tryptic soy agar (TSA) were purchased from Guangdong Huankai Microbial Sci. \& Tech. Co., Ltd. (Guangzhou, China).

\section{Synthesis of PAF26 peptide self-assembled hydrogel}

Firstly, $40 \mathrm{mg}$ PAF26 peptide was dissolved in $1 \mathrm{~mL}$ distilled water. Then, the $\mathrm{pH}$ was adjusted by adding $0.5 \mathrm{M} \mathrm{NaOH}$ solution in 5-10 $\mu \mathrm{L}$ increments. When the $\mathrm{pH}$ reached a value of 7.5 , the solution was kept at that $\mathrm{pH}$ for several minutes to form the self-assembled hydrogel, and then, the self-assembled hydrogel was stored at RT (for at least 1 day) for the following studies.

\section{Rheology test}

The rheological property of the self-assembled hydrogel was evaluated using a control-strain rheometer (Anton Paar MCR302, Austria). Frequency sweep test was conducted at a strain, which was within the linear viscoelastic region $(1 \%$ strain). The frequency was varied from 0.1 to $10 \mathrm{~Hz}$, and the temperature was set as $37^{\circ} \mathrm{C}$ (the temperature of the human body). Then, the storage modulus $\left(G^{\prime}\right)$ and loss modulus $\left(G^{\prime \prime}\right)$ were recorded. The viscosity of the self-assembled hydrogel was examined at the shear rate, which was varied from 0.1 to $1 \mathrm{~s}^{-1}$ at $25{ }^{\circ} \mathrm{C}$ (the temperature of injection).

\section{Scanning electron microscopy (SEM)}

The microscopic structure of the self-assembled hydrogel was evaluated by scanning electron microscopy. Once selfassembled into a hydrogel, the self-assembled hydrogel was placed in liquid nitrogen to freeze and then lyophilized by a freeze-dryer (LGJ-10, China). In addition, the self-assembled hydrogel, after being stored at room temperature for $24 \mathrm{~h}$, was also lyophilized. Then, the lyophilized hydrogels were observed under a scanning electron microscope (FEI Quanta 250 FEG, USA).

\section{Circular dichroism}

The solid circular dichroism of the self-assembled hydrogel was analyzed using a spectropolarimeter (JASCOJ-815, Japan), with accumulations of $4 \mathrm{~s}$ in every $1 \mathrm{~nm}$ in the wavelength range of 190-450 nm.

\section{Fourier transform infrared spectroscopy (FT-IR)}

$2 \mathrm{mg}$ of the lyophilized self-assembled hydrogel was mixed with 100-200 $\mathrm{mg}$ of $\mathrm{KBr}$. Then, the mixture was ground and repressed into a piece. The FTIR spectra were then collected on a spectrophotometer (Nicolet iS50, USA).

\section{Ultraviolet-visible absorbance spectroscopy}

A series of PAF26 peptide solutions at different concentrations: $0.2,0.5,1,2$, and $40 \mathrm{mg} \mathrm{mL}^{-1}$ were prepared, and the ultraviolet-visible absorbance spectra were recorded on an ultraviolet-visible spectrophotometer (UV1800PC, China). In addition, the ultraviolet-visible absorbance spectra of the selfassembled hydrogel were also recorded.

\section{Fluorescence spectroscopy}

A series of PAF26 peptide solutions at different concentrations: $0.2,0.5,1,2$, and $40 \mathrm{mg} \mathrm{mL^{-1 }}$ were prepared, and the fluorescence spectra were recorded on a fluorescence spectrophotometer (F-7100, Japan); $\lambda_{\mathrm{ex}}=310 \mathrm{~nm}, \lambda_{\mathrm{em}}=315-500 \mathrm{~nm}$. Moreover, the fluorescence spectra of the self-assembled hydrogel were also recorded.

\section{Antimicrobial activity evaluation}

Before antimicrobial experiments, the samples: self-assembled hydrogel and agar culture medium as control (for Candida albicans, SDA culture medium was chosen; for Staphylococcus aureus and Escherichia coli, TSA culture medium was chosen), were sterilized for $1 \mathrm{~h}$ using UV light.

To evaluate the antimicrobial activity, the pathogenic microbes: Candida albicans (fungus), Staphylococcus aureus (Gram-positive), and Escherichia coli (Gram-negative) were chosen. The microbial solutions were prepared in the following way: firstly, the Candida albicans was incubated in SDB culture medium at $26^{\circ} \mathrm{C}$, and the Staphylococcus aureus and Escherichia coli were incubated in TSB culture medium at $37{ }^{\circ} \mathrm{C}$. After incubating them overnight, $0.2 \mathrm{~mL}$ of Candida albicans, Staphylococcus aureus, and Escherichia coli microbial medium were each seeded in $30 \mathrm{~mL}$ fresh culture medium and further cultured. When the optical density (OD) value of the microbial solution at $600 \mathrm{~nm}$ reached 0.1 , the antimicrobial experiments were conducted as below.

Zone of inhibition test. $200 \mu \mathrm{L}$ of each microbial solution was plated on the agar dishes (covered with agar culture medium), and then, $100 \mu \mathrm{L}$ of the self-assembled hydrogel or agar culture medium was placed at the center of the agar dish. After culturing for $24 \mathrm{~h}$, the diameter of the zone of inhibition was measured for evaluating the antimicrobial activity.

Antimicrobial activity test. Firstly, $100 \mu \mathrm{L}$ of the selfassembled hydrogel or agar culture medium was transferred to a 96 -well plate. Then, $50 \mu \mathrm{L}$ of each microbial solution was planted on each sample. The microbial solution was further incubated for $6 \mathrm{~h}$, and the OD value at $600 \mathrm{~nm}$ was monitored on a microplate reader (DNM-9602, China) at an interval of $2 \mathrm{~h}$. To further evaluate the killing efficiency, $10 \mu \mathrm{L}$ of each microbial solution after $6 \mathrm{~h}$ incubation was suctioned and diluted to 
$200 \mu \mathrm{L}$, and then transferred on an agar dish. After culturing for $24 \mathrm{~h}$, the growth of the microbial colonies was imaged.

SEM observation. To image the morphology of the pathogenic microbes, after co-culturing the pathogenic microbes with self-assembled hydrogel or agar culture medium for $6 \mathrm{~h}$, the microbial solution was collected and centrifuged at $4000 \mathrm{rpm}$ for 5 min. ${ }^{31}$ They were then washed with PBS and fixed with 5\% formaldehyde for half an hour. The pathogenic microbes were further washed with distilled water, followed by washing several times with ethanol for dehydration. Afterward, the pathogenic microbes were dropped on cleaned silicon chips and air-dried at room temperature for SEM analysis. The images were recorded on SEM (FEI Quanta 250 FEG, USA).

\section{Results and discussion}

\section{Self-assembled molecular hydrogel}

PAF26 peptide is a hexapeptide with an amphipathic structure. After dissolving it in distilled water, an acidic and transparent solution of PAF26 was obtained. On the other hand, when the $\mathrm{pH}$ value was adjusted to 7.5, the solution was found to transform into a semi-transparent hydrogel (Fig. 1A). Storage moduli $\left(G^{\prime}\right)$ of the semi-transparent hydrogel was observed to be greater than the loss moduli $\left(G^{\prime \prime}\right)$ (Fig. 1B), which in turn confirmed that the self-assembled molecular hydrogel possessed the characteristic of a viscoelastic solid.

Further, the viscosity test found that the viscosity of the selfassembled molecular hydrogel significantly dropped with an increasing shear rate (Fig. 1C), thereby showing shear-thinning ability, which indicated that the self-assembled molecular hydrogel was injectable and could be used through a syringe for clinical applications. ${ }^{31}$

\section{Mechanism of the self-assembled hydrogel}

The self-assembly of the amphipathic peptide usually depends on the intermolecular forces and hydrophobic effect. The

A
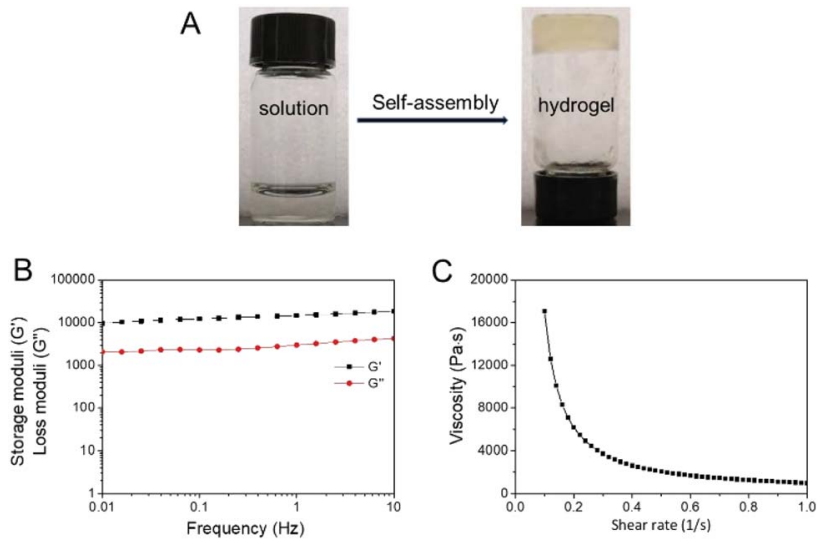

Fig. 1 (A) The image of PAF26 peptide transformation from solution to self-assembled hydrogel; (B) storage moduli $\left(G^{\prime}\right)$ and loss moduli $\left(G^{\prime \prime}\right)$ of the self-assembled hydrogel as a function of frequency from 0.1 to $10 \mathrm{~Hz}$ at $37{ }^{\circ} \mathrm{C}$; (C) viscosity of the self-assembled hydrogel as a function of shear rate from 0.1 to $1 \mathrm{~s}^{-1}$ at $25^{\circ} \mathrm{C}$.

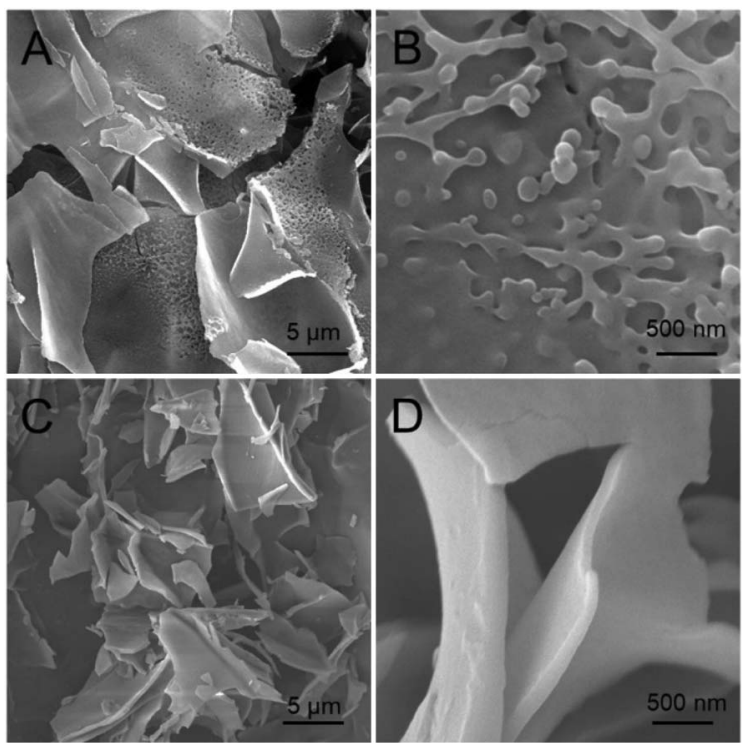

Fig. 2 Scanning electron microscopy (SEM) images of (A and B) the primarily formed self-assembled hydrogels after freeze-drying and ( $C$ and D) the self-assembled hydrogel after being stored at RT for $24 \mathrm{~h}$ and freeze-dried.

chemical structure of PAF26 peptide consists of three hydrophobic amino acids and three hydrophilic cationic amino acids. The SEM images showed that when primarily formed, the selfassembled hydrogel had a micro-sheet structure with nanoparticles on the surface (Fig. 2A and B), while after storing for $24 \mathrm{~h}$ at room temperature, the self-assembled hydrogel had a uniform micro-sheet structure (Fig. 2C and D).

Fourier transform infrared spectroscopy (FT-IR) and circular dichroism (CD) were conducted to evaluate the secondary structure transformation of the self-assembled peptide. According to the FT-IR spectrum (Fig. 3A), the adsorption band of amide-I at $1633 \mathrm{~cm}^{-1}$ seemed to be the characteristic absorbance of the $\beta$-sheet conformation; the adsorption band of amide-II at $1677 \mathrm{~cm}^{-1}$ indicated the $\beta$-sheet or $\beta$-turn conformation structure of the self-assembled hydrogel. ${ }^{32}$ In addition, the adsorption band of $\mathrm{N}-\mathrm{H}$ at $3290 \mathrm{~cm}^{-1}$ confirmed that the peptide was self-assembled via intermolecular backbonebackbone hydrogen bonding. In the solid circular dichroism spectrum (Fig. 3B), a strong positive peak was found at $195 \mathrm{~nm}$, and a broad negative peak was found at $230 \mathrm{~nm}$, confirming the $\beta$-sheet structure of the self-assembled hydrogel, while the
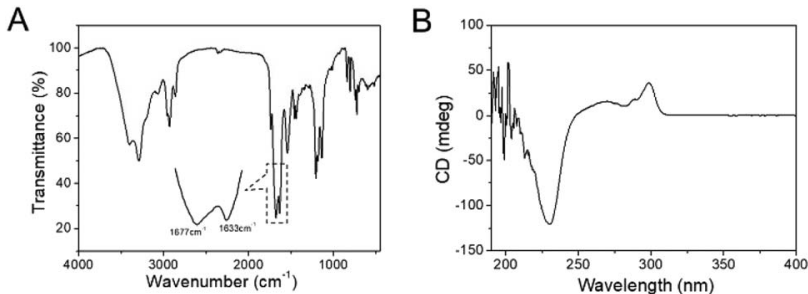

Fig. 3 (A) FT-IR spectra of the freeze-dried self-assembled hydrogel; (B) the circular dichroism (CD) spectra of the self-assembled hydrogel. 

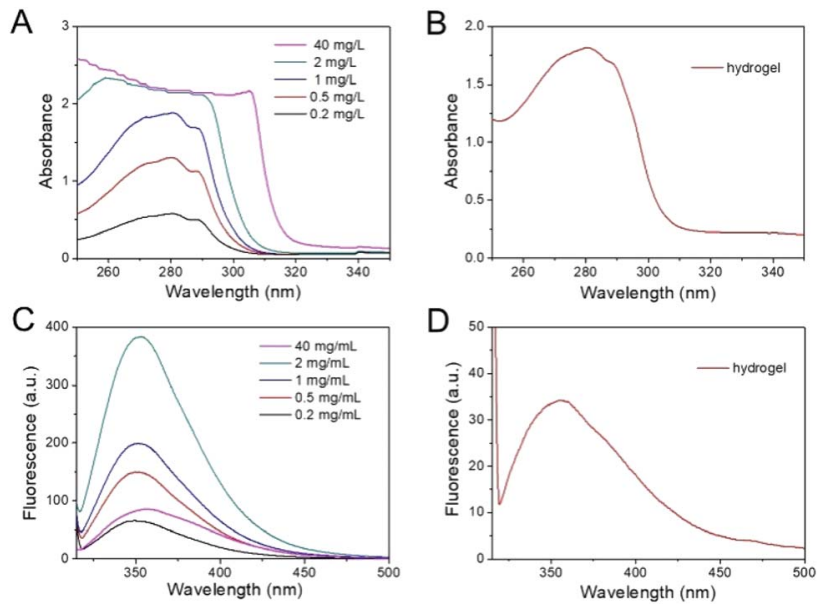

Fig. 4 The UV-vis absorbance spectra (A and B) and fluorescence emission $\left(\lambda_{\text {ex }}=310 \mathrm{~nm}\right.$ ) spectra $(C$ and $D)$ : $(A$ and $C)$ PAF26 solutions at different concentrations: $0.2,0.5,1,2$, and $40 \mathrm{mg} \mathrm{mL}$; (B and $D)$ the self-assembled hydrogel.

negative peak at $199 \mathrm{~nm}$ confirmed that there is still some random coil in the hydrogel.

The UV-vis absorbance and fluorescence emission spectra were used to evaluate the effect of aromatic-aromatic $\pi-\pi$ stacking interactions in the self-assembly of the PAF26 peptide. When the concentration of PAF26 peptide was increased from 0.2 to $1 \mathrm{mg} \mathrm{mL} \mathrm{m}^{-1}$, the maximum absorption peak was only subtly shifted (Fig. 4A), while at $2 \mathrm{mg} \mathrm{mL}^{-1}$ and $40 \mathrm{mg} \mathrm{mL}^{-1}$ (though at $40 \mathrm{mg} \mathrm{mL} \mathrm{m}^{-1}$, due to the high concentration, the absorbance from 250-310 nm could not make sense), the maximum absorption peak was red-shifted, thereby confirming that upon reaching a higher concentration, the PAF26 peptide could interact via aromatic-aromatic $\pi-\pi$ stacking interactions. $^{33}$ Further, the UV-vis absorbance spectra of the selfassembled hydrogel confirmed a red-shift of the maximum absorption peak in the self-assembled hydrogel (Fig. 4B). On the other hand, in the fluorescence spectra, the central peak shifted from 350 to $360 \mathrm{~nm}$ with an increase in the concentration of PAF26 peptide from 0.2 to $1 \mathrm{mg} \mathrm{mL}^{-1}$ (Fig. 4C). Similar to the PAF26 peptide, the central peak of the self-assembled hydrogel also displayed an obvious red-shift to $360 \mathrm{~nm}$ (Fig. 4D).

As shown in Scheme 2, it could be supposed that when the $\mathrm{pH}$ value shifted to 7.5, the PAF26 peptide was forced by aromatic-aromatic $\pi-\pi$ stacking interactions to have a secondary structure of $\beta$-sheet, which formed the selfassembled micelles, and then the micelles got linked to the catenulate microfiber, polymerized to microsheet, and finally formed the self-assembled hydrogel.

\section{Antimicrobial activity study}

The antimicrobial activities of the self-assembled hydrogel were evaluated against various pathogenic microbes such as Candida albicans, Staphylococcus aureus, and Escherichia coli. When introduced to microbial culture dishes, the self-assembled hydrogel could inhibit the growth of Candida albicans, Staphylococcus aureus, and Escherichia coli in situ (Fig. 5A-C).
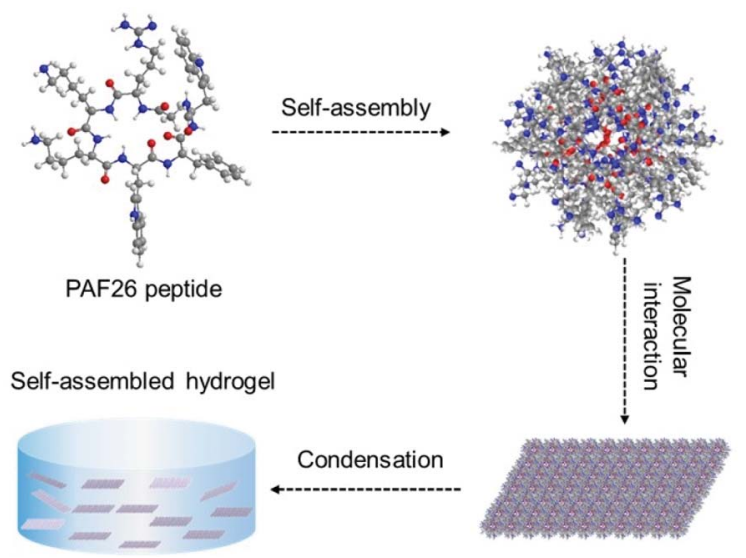

Scheme 2 The proposed self-assembly mechanism of PAF26 peptide to form the hydrogel.

Meanwhile, the hydrogel could permeate and inhibit microbial growth even outside. For Candida albicans, the diameter of the inhibition zone was $2.00 \pm 0.06 \mathrm{~cm}$; for Staphylococcus aureus, the diameter of the inhibition zone was $1.53 \pm$ $0.21 \mathrm{~cm}$; and for Escherichia coli, the diameter of the inhibition zone was $2.01 \pm 0.06 \mathrm{~cm}$. On the contrary, as a control, the agar culture medium could hardly inhibit the microbial growth (Fig. 5D-F).

Besides, Fig. 6A-C showed that when co-cultured, the optical densities of Candida albicans, Staphylococcus aureus, and Escherichia coli on an agar culture medium were increased with an increase in the incubation time, which confirmed that the pathogenic microbes were in good condition. Whereas, in the case of the self-assembled hydrogel, the optical densities exhibited almost no change, thereby demonstrating that the self-assembled hydrogel could suppress the microbial growth. Moreover, after being co-cultured with the self-assembled hydrogel, no obvious microbial colonies were found on the dishes, which established that the killing efficiency of the hydrogel was about 100\% (Fig. 6D-F).
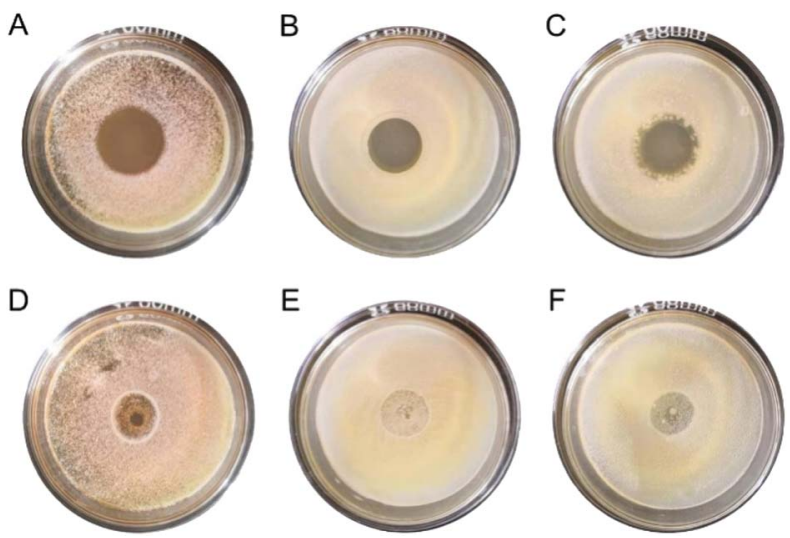

Fig. 5 Zone of antimicrobial inhibition against Candida albicans ( $\mathrm{A}$ and D), Staphylococcus aureus (B and E) and Escherichia coli ( $C$ and F) after being cultured for $24 \mathrm{~h}$ : $(A-C)$ self-assembled hydrogel, and $(D-F)$ agar culture medium. 
A
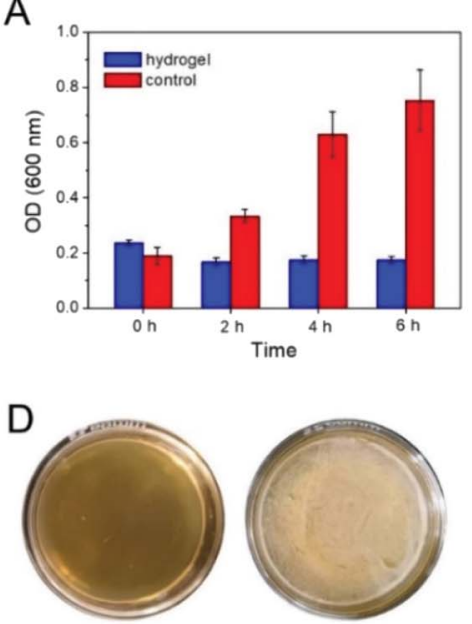

hydrogel

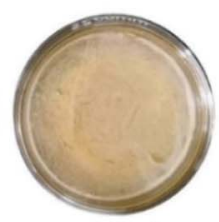

control
B

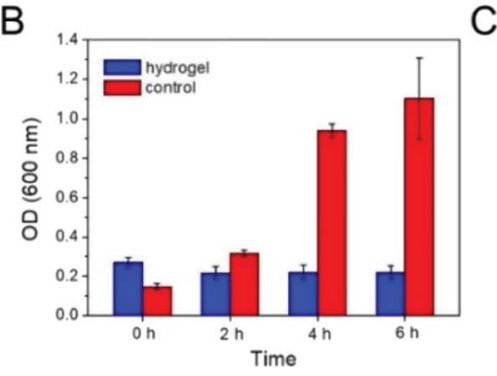

E

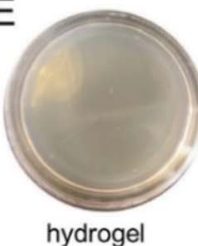

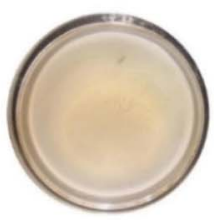

control

\section{C}
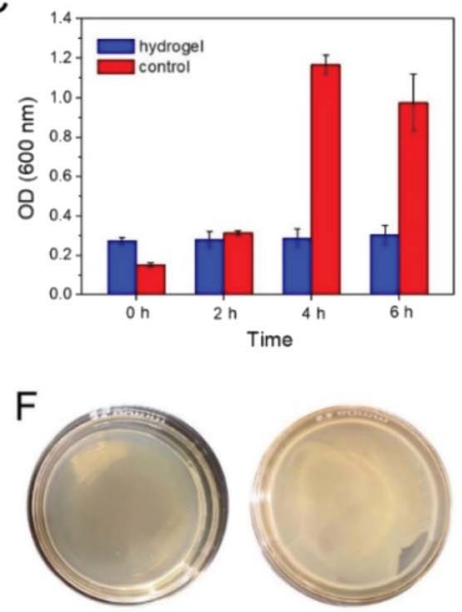

hydrogel

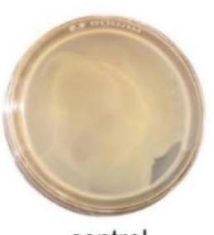

control

Fig. 6 ( $\mathrm{A}-\mathrm{C}$ ) $O \mathrm{D}_{600 \mathrm{~nm}}$ values during $6 \mathrm{~h}$ culture of the self-assembled hydrogel and agar culture medium (as control) with Candida albicans (A), Staphylococcus aureus (B), and Escherichia coli (C). (D-F) The images of the microbial colonies after $24 \mathrm{~h}$ culture on agar dishes: Candida albicans (D), Staphylococcus aureus (E), and Escherichia coli (F).
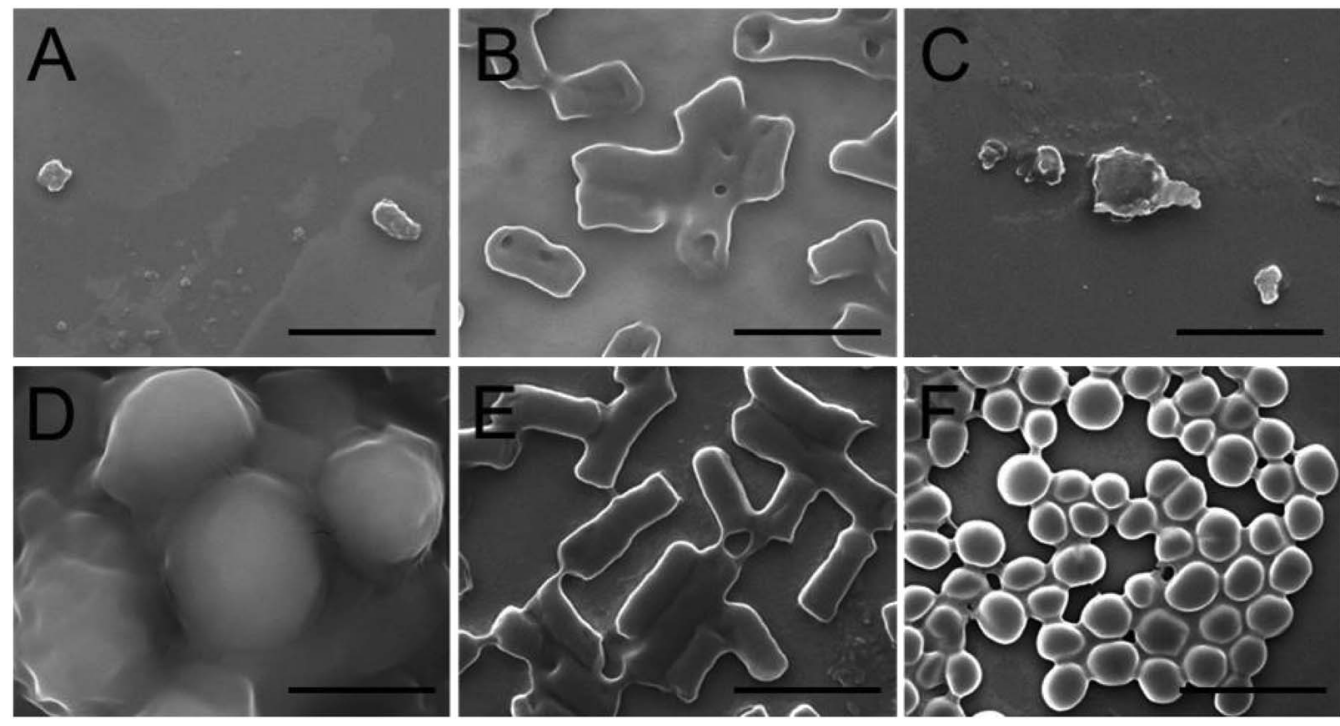

Fig. 7 Scanning electron microscopy (SEM) images of Candida albicans (A and D), Staphylococcus aureus (B and E) and Escherichia coli (C and F) after being incubated with ( $A-C)$ self-assembled hydrogel or ( $D$ and $E$ ) agar culture medium for $6 \mathrm{~h}$. The scale bars are $2 \mu \mathrm{m}$.

The mechanism of the antimicrobial effect was supposed to be the destruction of the cell membrane. Therefore, the morphologies of Candida albicans, Staphylococcus aureus, and Escherichia coli were investigated by SEM. As shown in Fig. 7, the microbial cells treated with an agar culture medium as control were smooth and retained their respective morphologies: Candida albicans and Staphylococcus aureus exhibited a round shape, and Escherichia coli showed a rod-like shape. In contrast, the serious surface roughness and shape changes were observed for the microbial cells exposed to the selfassembled hydrogel, thereby supporting that the antimicrobial effect of the hydrogel was due to its interaction with the microbial cell wall.

\section{Conclusions}

In this work, the self-assembled hydrogel was synthesized from amphipathic PAF26 peptide, with the synthesis being triggered by $\mathrm{pH}$. The self-assembly was forced by the hydrophobicity and aromatic-aromatic $\pi-\pi$ stacking interactions of the PAF26 peptide to form $\beta$-sheet conformations, thereby resulting in a micro-sheet structure. In addition, the self-assembled hydrogel showed shear-thinning ability and was injectable. Further, the antimicrobial studies confirmed that the self-assembled hydrogel possessed good antimicrobial potential against fungus, Gram-positive and Gram-negative pathogenic microbes. Thus, this study gives a novel method for the application of antimicrobial peptide for antimicrobial therapies. 


\section{Conflicts of interest}

There are no conflicts to declare.

\section{Acknowledgements}

This work was financially supported by the National Natural Science Foundation of China (No. 51703260, 51703259), and Program for Science and Technology of Henan Province (No. 182102310635).

\section{Notes and references}

1 A. P. Gomes, J. F. Mano, J. A. Queiroz and I. C. Gouveia, Carbohydr. Polym., 2015, 127, 451.

2 Y. Yuan, H. Wu, H. F. Lu, Y. R. Zheng, J. Y. Ying and Y. G. Zhang, Chem. Commun., 2019, 55, 699.

3 C. G. Otoni, M. R. de Moura, F. A. Aouada, G. P. Camilloto, R. S. Cruz, M. V. Lorevice, N. de F. F. Soares and L. H. C. Mattoso, Food Hydrocolloids, 2014, 41, 188.

4 A. H. León, F. Hebal, C. Stake, K. Baldwin and K. A. Barsness, Int. Wound J., 2019, 16, 41.

5 P. Li, Y. F. Poon, W. F. Li, H. Y. Zhu, S. H. Yeap, Y. Cao, X. B. Qi, C. C. Zhou, M. Lamrani, R. W. Beuerman, E. T. Kang, Y. G. Mu, C. M. Li, M. W. Chang, S. S. J. Leong and M. B. Chan-Park, Nat. Mater., 2011, 10, 149.

6 S. D. Mu, W. T. Liu, L. Zhao, Y. R. Long and H. B. Gu, Polymer, 2019, 169, 80.

7 X. Y. Qiu, P. Y. Yuan, K. Lei, L. Wang, Y. K. Bai, S. Y. Liu and X. Chen, ACS Appl. Mater. Interfaces, 2018, 10, 17018.

8 W. R. Leow, X. Liu, Y. L. Wu, P. Z. Guo, X. J. Loh and X. D. Chen, Chem. Soc. Rev., 2018, 47, 6917.

9 Y. Zhang, J. H. Zhang, M. Chen, H. Gong, S. Thamphiwatana, L. Eckmann, W. W. Gao and L. F. Zhang, ACS Appl. Mater. Interfaces, 2016, 8, 18367.

10 L. Wang, X. Li, T. Sun, Y. H. Tsou, H. Chen and X. Y. Xu, Macromol. Biosci., 2018, 18, 1700325.

11 Y. C. Guo, S. Wang, H. H. Du, X. L. Chen and H. Fei, Biomacromolecules, 2018, 20, 558.

12 I. A. Neacsu, L. V. Arsenie, R. Trusca, I. L. Ardelean, N. Mihailescu, I. N. Mihailescu, C. Ristoscu, C. Bleotu, A. Ficai and E. Andronescu, Nanomaterials, 2019, 9, 692.

13 P. Li, Z. P. Feng, Z. Y. Yu, Y. Chen, P. W. Li, Z. M. Yang, S. D. Li and S. H. Jin, Int. J. Biol. Macromol., 2019, 133, 67.
14 D. A. Salick, J. K. Kretsinger, D. J. Pochan and J. P. Schneider, J. Am. Chem. Soc., 2007, 129, 14793.

15 H. Sun, Y. X. Hong, Y. J. Xi, Y. J. Zou, J. Y. Gao and J. Z. Du, Biomacromolecules, 2018, 19, 1701.

16 M. A. Fox, J. E. Thwaite, D. O. Ulaeto, T. P. Atkins and H. S. Atkins, Peptides, 2012, 33, 197.

17 K. Hilpert, R. Volkmer-Engert, T. Walter and R. E. Hancock, Nat. Biotechnol., 2005, 23, 1008.

18 V. W. L. Ng, J. M. W. Chan, H. Sardon, R. J. Ono, J. M. García, Y. Y. Yang and J. L. Hedrick, Adv. Drug Delivery Rev., 2014, 78, 46.

19 H. Wang and Z. Yang, Nanoscale, 2012, 4, 5259.

20 F. Zhao, M. L. Ma and B. Xu, Chem. Soc. Rev., 2009, 38, 883. 21 B. G. Xing, C. W. Yu, K. H. Chow, P. L. Ho, D. G. Fu and B. Xu, J. Am. Chem. Soc., 2002, 124, 14846.

22 J. L. Li, R. R. Xing, S. Bai and X. H. Yan, Soft Matter, 2019, 15, 1704.

23 H. L. Jin, C. Wan, Z. Zou, G. F. Zhao, L. L. Zhang, Y. Y. Geng, T. Chen, A. Huang, F. G. Jiang, J. P. Feng, J. F. Lovell, J. Chen, G. Wu and K. Y. Yang, ACS Nano, 2018, 12, 3295.

24 L. Y. Zhao, Q. L. Zou and X. H. Yan, Bull. Chem. Soc. Jpn., 2019, 92, 70.

25 K. Fukunaga, H. Tsutsumi and H. Mihara, Bull. Chem. Soc. Jpn., 2019, 92, 391.

26 S. Akama, T. Maki and M. Yamanaka, Chem. Commun., 2018, 54, 8814.

27 P. Xing, H. Chen, H. Xiang and Y. L. Zhao, Adv. Mater., 2018, 30, 1705633.

28 Z. M. Yang, K. M. Xu, L. Wang, H. W. Gu, H. Wei, M. J. Zhang and B. Xu, Chem. Commun., 2005, 4414.

29 A. Muñoz, M. Gandaí, E. Harries, L. Carmona, N. D. Read and J. F. Marcos, Fungal Biol. Rev., 2013, 26, 146.

30 L. Mendive-Tapia, C. Zhao, A. R. Akram, S. Preciado, F. Albericio, M. Lee, A. Serrels, N. Kielland, N. D. Read, R. Lavilla and M. Vendrell, Nat. Commun., 2016, 7, 10940.

31 Y. Li, K. Fukushima, D. J. Coady, A. C. Engler, S. Q. Liu, Y. Huang, J. S. Cho, Y. Guo, L. S. Miller, J. P. Tan, P. L. Ee, W. M. Fan, Y. Y. Yang and J. L. Hedrick, Angew. Chem., Int. Ed., 2013, 52, 674-678.

32 S. Y. Qin, H. F. Jiang, X. J. Liu, Y. Pei, H. Cheng, Y. X. Sun and X. Z. Zhang, Soft Matter, 2014, 10, 947.

33 S. Y. Qin, S. S. Xu, R. X. Zhuo and X. Z. Zhang, Langmuir, 2011, 28, 2083. 